\title{
NetPot: Easy Meal Enjoyment for Distant Diners
}

\author{
Zoltan Foley-Fisher, Vincent Tsao, Johnty Wang, and Sid Fels \\ Department of Electrical and Computer Engineering, University of British Columbia, \\ Vancouver, Canada \\ $\{$ zoltan, vtsao, johnty, ssfels\}@ece.ubc.ca
}

\begin{abstract}
We capture key factors of a group meal with communication and interface technologies to make a meal more enjoyable for diners who cannot be collocated. We determined three factors of a popular group meal, Chinese hotpot, that are essential for a group meal experience: interacting as a group with food, a central shared hotpot, and a feeling that others are nearby. We developed a prototype system to maintain these factors for an online meal with remote friends. Our technique is of interest to designers creating technology for isolated diners.
\end{abstract}

Keywords: Augmented Reality, Hotpot, Online Meals, Social Networking.

\section{Introduction}

Family or friends often enjoy meals together, but sometimes not everyone can join the meal. This is particularly true as older people live independently, younger people live alone [1] and working people travel more and work from afar. In these cases, one or all the group has to eat alone - an experience that is not as enjoyable as a group meal.

Ubiquitous, high bandwidth and inexpensive communications technology offer a solution. Telepresence technologies [2], like Skype, may have interaction limited to audio and grainy video, but we can build on these technologies to create a system that captures the factors of an enjoyable group meal so that remote diners can join the fun.

To our knowledge, we are the first researchers to use targeted interactions to focus only on communicating important factors of group meals with simple augmented-reality technologies rather than trying to recreate a highly realistic tele-eating experience. Maintaining the factors with our prototype ultimately improved diner's enjoyment when sharing a hotpot meal with remote friends. Our system can be made cheaply and is easy to set up. Until truly realistic telepresence technologies are available, our approach may well be the most enjoyable way to eat with remote friends.

\section{Missing Meal Factors}

In our study, we focussed on Chinese hotpot. Hotpot is a communal meal eaten in East Asia, and is popular among Canadian-Asian communities in Vancouver. In hotpot, a group of friends or family members simmers a central pot of stock at the dining table 
where each person uses their chopsticks to select items to cook from an assortment of plates of food around the table such as sliced meat, vegetables and seafood. Hotpot is a social meal that takes a long time to eat and is often enjoyed on special occasions. We surveyed diners and observed three factors to be important in this meal:

1. Interacting as a group with food. Some diners adopt roles, such as the person in charge of putting in specific foods. There is often a formal order when food is taken out of the pot. Some diners test the food to tell others if it is cooked.

2. Central shared hotpot. Hotpot diners all share the same pot. Diners rarely turn away from the hotpot. The meal lasts a long time. Even when talking to others, diners focus their attention on the food in the pot.

3. Company, a feeling that others are nearby. Even though diners do not pay a lot of attention to others, they are nevertheless aware of them in their periphery.

By maintaining these factors, we expect that meals with remote diners can be made more enjoyable. We also aim to select the least complex and expensive technologies that will result in significant improvements to meal enjoyment. Teleconferencing software, such as Skype, cannot recover all the missing factors alone. We developed low-fidelity prototypes to determine that designs to recover meal factors should: Adopt avatars to represent remote diners, Acknowledge that diners are not concerned with what remote diners are eating, Involve simple interactions, Use readily available and inexpensive components, and Make sure audio is included.

\section{Prototype}

For our prototype, half of a traditional Yuān Yāng Gū̄, or "Mandarin Duck", hotpot is used for cooking, while the other half serves as a display and tracking surface to interact with remote diners. The interactive surface of the pot is a white space on which images are projected, and the movements of the diner's chopsticks within this space are tracked with a video camera. An induction stove is used as a safe and compact heating element. This is shown in Fig 1.

Up to three virtual food items, such as gailan (a type of broccoli), fish balls and tofu, appear on the interaction surface as if dropped into the pot. The food floats around as it slowly gets "cooked". The food initially appears raw (meats are red/pink in color), and changes to a cooked appearance as it stays in the pot. If food has been in the pot too long, it appears "overcooked". A diner can move a piece of food by placing the tip of the chopstick on it, and then moving the chopsticks. For simplicity, the food cannot be dropped once picked up, but only placed into a "container".

Three diners are represented by avatars at the edge of the interactive surface. A diner is fed when food moves close to their avatar. Each diner has a "satisfaction bar" that is incremented each time they are fed. Cooked food increments the bar, overcooked food decrements the bar. Diners are notified of the names of remote diners who feed their avatar. The avatar enters a sleep state if remote diners don't interact with their hotpot.

The system augments a typical hotpot arrangement with a computer, overhead projector and webcam to track the chopsticks and runs over the internet using our software and Skype ${ }^{\mathrm{TM}}$. 


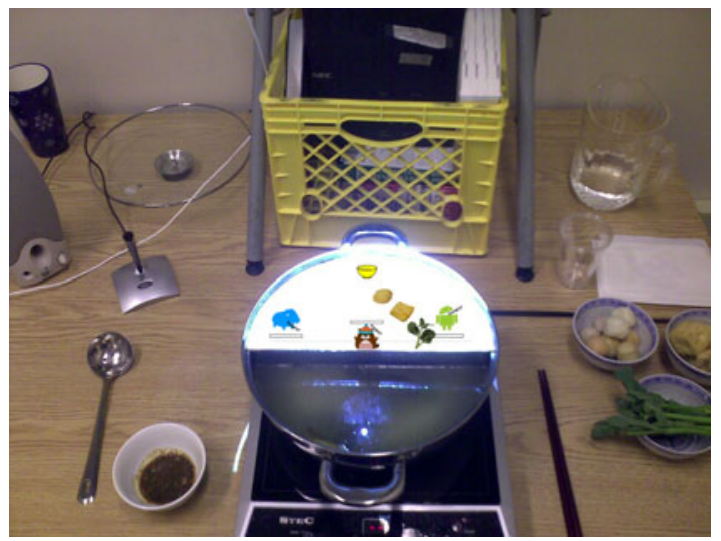

Fig. 1. Inductive hot-plate and the NetPot system of projected remote diner actions, chopstick tracking cameras and Skype audio teleconferencing

\section{Conclusion}

We determined three meal factors missing when diners eat Chinese hotpot alone. We assumed that if these factors were maintained for a tele-eating experience, then diner's meals would be more enjoyable without trying to recreate the exact meal experience using high-end teleconferencing. We targeted interaction designs with readily available augmented reality technology to keep the missing factors in an online meal with remote friends.

We evaluated a prototype of the design - NetPot - to assess how much more enjoyable the meal is than when sharing the meal with a low quality telepresence system. We maintained the meal factors, although not completely. The meal was more enjoyable, but it is not clear if it was because the factors were there or because the interactions we chose were enjoyable.

Until telepresence systems provide complete realism and are affordable, a careful treatment of the important meal factors for remote diners with targeted interactions may lead to more enjoyable shared dining even when friends and family cannot be together.

\section{References}

1. Sellaeg, K., Chapman, G.E.: Masculinity and food ideals of men who live alone. Appetite 51(1), 120-128 (2008), doi:10.1016/j.appet.2008.01.003

2. Sheridan, T.B.: Musings on telepresence and virtual presence. Presence: Teleoperators and Virtual Environments 1(1) (1992), http://portal.acm.org/citation.cfm?id $=128956$ 